\title{
Éric DESROSIERS
}

Journaliste, Le Devoir, Montréal.

\author{
(25 février 2010)
}

\section{"Le débat sur les finances publiques est en proie à des «mythes». Le problème de la dette est grandement exagéré, clame un collectif."}

\author{
Un document produit en version numérique par Jean-Marie Tremblay, bénévole, \\ professeur de sociologie au Cégep de Chicoutimi \\ Courriel: jean-marie tremblay@uqac.ca \\ Site web pédagogique : http://www.uqac.ca/jmt-sociologue/ \\ Dans le cadre de: "Les classiques des sciences sociales" \\ Une bibliothèque numérique fondée et dirigée par Jean-Marie Tremblay, \\ professeur de sociologie au Cégep de Chicoutimi \\ Site web: http://classiques.uqac.ca/ \\ Une collection développée en collaboration avec la Bibliothèque \\ Paul-Émile-Boulet de l'Université du Québec à Chicoutimi \\ Site web: http://bibliotheque.uqac.ca/
}




\section{Politique d'utilisation de la bibliothèque des Classiques}

Toute reproduction et rediffusion de nos fichiers est interdite, même avec la mention de leur provenance, sans l'autorisation formelle, écrite, du fondateur des Classiques des sciences sociales, Jean-Marie Tremblay, sociologue.

Les fichiers des Classiques des sciences sociales ne peuvent sans autorisation formelle:

- être hébergés (en fichier ou page web, en totalité ou en partie) sur un serveur autre que celui des Classiques.

- servir de base de travail à un autre fichier modifié ensuite par tout autre moyen (couleur, police, mise en page, extraits, support, etc...),

Les fichiers (.html, .doc, .pdf, .rtf, .jpg, .gif) disponibles sur le site Les Classiques des sciences sociales sont la propriété des Classiques des sciences sociales, un organisme à but non lucratif composé exclusivement de bénévoles.

Ils sont disponibles pour une utilisation intellectuelle et personnelle et, en aucun cas, commerciale. Toute utilisation à des fins commerciales des fichiers sur ce site est strictement interdite et toute rediffusion est également strictement interdite.

L'accès à notre travail est libre et gratuit à tous les utilisateurs. C'est notre mission.

Jean-Marie Tremblay, sociologue

Fondateur et Président-directeur général, LES CLASSIQUES DES SCIENCES SOCIALES. 
Cette édition électronique a été réalisée par Jean-Marie Tremblay, bénévole, professeur de sociologie au Cégep de Chicoutimi à partir de :

Éric Desrosiers, journaliste, Le Devoir

"Le débat sur les finances publiques est en proie à des «mythes». Le problème de la dette est grandement exagéré, clame un collectif”.

Le Devoir, Montréal, édition du jeudi, le 25 février 2010, pages B1 et B4. — Section Économie.

Polices de caractères utilisée :

Pour le texte: Times New Roman, 12 points.

Pour les citations : Times New Roman, 12 points.

Pour les notes de bas de page : Times New Roman, 12 points.

Édition électronique réalisée avec le traitement de textes Microsoft Word 2008 pour Macintosh.

Mise en page sur papier format : LETTRE (US letter), 8.5’’ x 11’’)

Édition numérique réalisée le 25 février 2010 à Chicoutimi, Ville de Saguenay, province de Québec, Canada. 


\section{Éric Desrosiers, journaliste, Le Devoir} Journaliste, Le Devoir

\section{"Le débat sur les finances publiques est en proie à des «mythes». Le problème de la dette est grandement exagéré, clame un collectif.”}

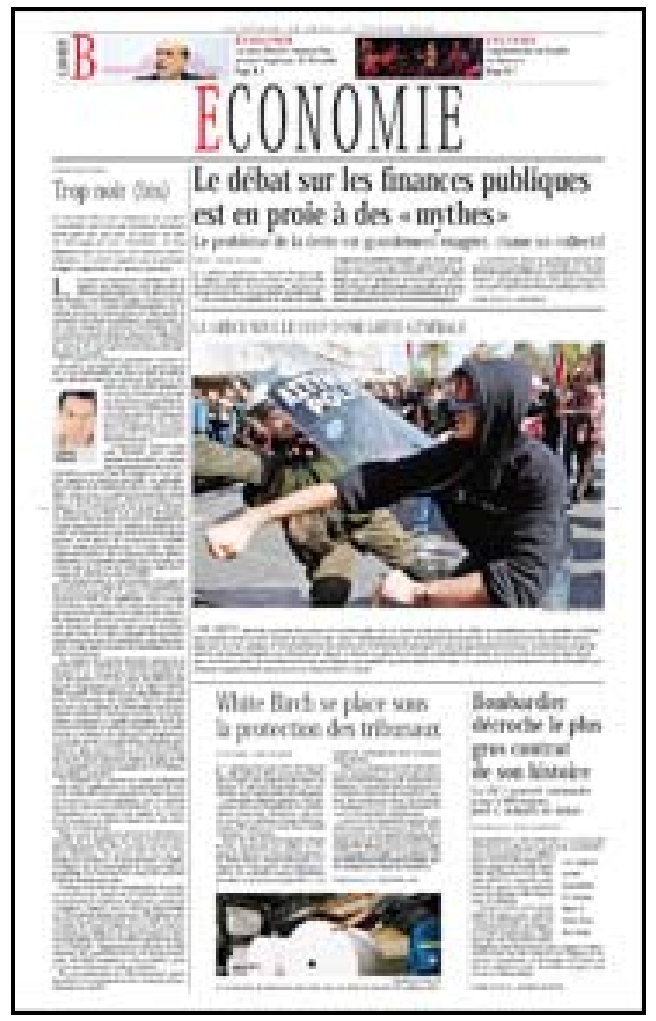

Le Devoir, Montréal, édition du jeudi, le 25 février 2010, pages B1 et B4. — Section Économie. 
Éric Desrosiers, journaliste, Le Devoir

"Le débat sur les finances publiques est en proie à des «mythes». Le problème de la dette est grandement exagéré, clame un collectif”.

Le Devoir, Montréal, édition du jeudi, le 25 février 2010, pages B1 et B4. — Section Économie.

Le débat actuel sur l'état des finances publiques québécoises se base sur « une vision étroite et biaisée » de l'économie, dénonce un groupe formé d'une cinquantaine d'intellectuels. "Le soi-disant problème de la dette du Québec m'apparaît grandement exagéré, a dit cette semaine au cours d'un entretien téléphonique au Devoir l'économiste Bernard Élie. La pire hypothèque que l'on semble en voie de laisser à nos enfants n'est pas celle de la dette, mais celle de services publics et de mécanismes de redistribution de la richesse rapetissés et d'un environnement dégradé.» Le professeur associé au Département des sciences économiques de l'UQAM est l'un des 49 intellectuels québécois, dont plusieurs économistes, qui joignent leur voix pour proposer «une autre lecture des finances publiques» dans le site Internet «Économie autrement» (http://www.economieautrement.org). Ils y dénoncent les nombreux «mythes » qui auraient cours dans ce débat, tel que le fait que l'endettement soit nécessairement une mauvaise chose pour les nations, que le secteur privé soit toujours plus efficace que le public ou encore que les économistes puissent exprimer des opinions neutres dans un tel débat.

Ils s'en prennent notamment aux recommandations des économistes du comité consultatif du ministre des Finances, Raymond Bachand, qui dévoilaient lundi un troisième et dernier fascicule dans lequel ils pressaient le gouvernement de recourir de manière égale à des réductions de dépenses et à des augmentations de taxes à la consommation et de tarifs afin de combler un déficit prévu de 11 milliards en 2013-2014. "Le mandat qui avait été confié [aux quatre experts] était trop contraignant. N'importe qui serait arrivé à la même conclusion », commence par répondre Bernard Élie. Les trois rapports trahissent cependant chez leurs auteurs " une vision unidimensionnelle très à court terme », ajoute-t-il quelques minutes plus tard. 


\section{Crise? Quelle crise?}

En ce qui concerne la dette, elle est pour le moment essentiellement attribuable à la crise économique et à l'erreur du gouvernement Charest de baisser les impôts et de ne pas récupérer les points de la TPS libérés par Ottawa, dit-il. Quant à celle que l'on voit continuer de grossir en même temps que vieillira la population québécoise, elle se fonde sur des projections à long terme qui ne sont pas plus fiables que celles des démographes qui n’ont pas vu venir le récent sursaut de fécondité des Québécois. On néglige aussi de souligner qu’une par tie de cette dette a, par exemple, servi à payer des salaires de chercheurs et de professeurs de cégep et d'université qui devraient compter comme un investissement dans les infrastructures de développement économique, au même titre que les ponts et les autoroutes.

Quoi qu'il en soit, " on n'est pas en face d'une dette monstrueuse. Ce n'est pas la Grèce », af firme Bernard Élie. Après tout, les pays développés ont déjà été bien plus endettés encore, au sortir de la Deuxième Guerre mondiale, et cela ne les a pas empêchés de connaître ensuite des décennies de prospérité.

\section{Le choix des Québécois}

De plus, c’est par choix que les dépenses publiques sont plus élevées au Québec qu'ailleurs en Amérique du Nord, rappelle le collectif Économie autrement, auquel se sont associés aussi, entre autres, les économistes Louis Gill et Gilles Dostaler, le professeur d'administration Omar Aktouf et les sociologues Gilles Bourque et Louise Vandelac. Si la population québécoise se contentait du même niveau de dépenses par habitant qu'en Ontario, leur gouvernement aurait 17,5 milliards de plus dans ses coffres.

Le comité d'experts du ministre Bachand rêve en couleur, dit-on, s’il pense que l'on peut trouver encore assez de gras à couper et de gains de productivité à réaliser dans le secteur public pour faire la moitié du chemin vers un budget équi- 
libré à l'horizon de 2013-2014. « L'essentiel des dépenses de l'État va en salaires en santé et en éducation, rappelle Bernard Élie. Qu'est-ce qu'on veut faire ? Couper dans le nombre d'enseignants et d'infirmières comme l'a fait Lucien Bouchard dans le temps du déficit zéro ? »

Cette idée d'introduire plus de concurrence du privé dans le secteur public pour en améliorer la productivité lui apparaît comme une autre imposture. La meilleure façon d'améliorer la productivité, dans le secteur public comme ailleurs, est d'avoir une main-d'oeuvre qualifiée, dit l'économiste. «De ce point de vue, le drame du décrochage scolaire me semble un problème bien plus grave que celui de la dette. »

\section{Des impôts, pas des tarifs}

«Ce sont les revenus de l'État qui sont trop bas et non les dépenses qui sont trop élevées », écrit le collectif Économie autrement. Mais pour augmenter ces revenus, pas question de recourir à des hausses de taxes à la consommation et de tarifs, comme le recommande le comité d'experts du ministre Bachand. Ces formes de taxation sont beaucoup trop régressives, dit-on, même si Bernard Élie convient que des mécanismes peuvent être mis en place pour en réduire les effets négatifs sur les ménages les plus modestes.

On voudrait plutôt que le gouvernement relève le niveau de ses impôts sur le revenu tout en leur donnant une structure plus progressive. Les seuls taxes et tarifs que l'on est prêts à voir augmenter sont ceux des «produits énergivores ou de luxe ». Il faudrait aussi tirer de meilleures redevances des ressources naturelles.

«Toutes ces questions exigent un débat beaucoup plus approfondi que celui que nous avons eu jusqu'à présent », conclut Bernard Élie.

\section{Le Devoir}

